\title{
COMPOSITE COTRIPLES AND DERIVED FUNCTORS
}

\author{
MICHAEL BARR
}

\section{Introduction}

The main result of [Barr (1967)] is that the cohomology of an algebra with respect to the free associate algebra cotriple can be described by the resolution given by U. Shukla in [Shukla (1961)]. That looks like a composite resolution; first an algebra is resolved by means of free modules (over the ground ring) and then this resolution is given the structure of a $D G$-algebra and resolved by the categorical bar resolution. This suggests that similar results might be obtained for all categories of objects with "two structures". Not surprisingly this turns out to involve a coherence condition between the structures which, for ordinary algebras, turns out to reduce to the distributive law. It was suggested in this connection by J. Beck and H. Appelgate.

If $\alpha$ and $\beta$ are two morphisms in some category whose composite is defined we let $\alpha \cdot \beta$ denote that composite. If $S$ and $T$ are two functors whose composite is defined we let $S T$ denote that composite; we let $\alpha \beta=\alpha T^{\prime} \cdot S \beta=S^{\prime} \beta \cdot \alpha T: S T \rightarrow S^{\prime} T^{\prime}$ denote the natural transformation induced by $\alpha: S \rightarrow S^{\prime}$ and $\beta: T \rightarrow T^{\prime}$. We let $\alpha X: S X \rightarrow S^{\prime} X$ denote the $X$ component of $\alpha$. We let the symbol used for an object, category or functor denote also its identity morphism, functor or natural transformation, respectively. Throughout we let $\mathfrak{M}$ denote a fixed category and $\mathfrak{A}$ a fixed abelian category. $\mathfrak{N}$ will denote the category of simplicial $\mathfrak{M}$ objects (see 1.3. below) and $\mathfrak{B}$ the category of cochain complexes over $\mathfrak{A}$.

\section{Preliminaries}

In this section we give some basic definitions that we will need. More details on cotriples may be found in [Barr \& Beck (1966)], [Beck (1967)] and [Huber (1961)]. More details on simplicial complexes and their relevance to derived functors may found in [Huber (1961)] and [Mac Lane (1963)].

Definition 1.1. A cotriple $\mathbf{G}=(G, \varepsilon, \delta)$ on $\mathfrak{M}$ consists of a functor $G: \mathfrak{M} \rightarrow \mathfrak{M}$ and natural transformations $\varepsilon: G \rightarrow \mathfrak{M}$ and $\delta: G \rightarrow G^{2}(=G G)$ satisfying the identities $\varepsilon G \cdot \delta=G \varepsilon \cdot \delta=G$ and $G \delta \cdot \delta=\delta G \cdot \delta$. From our notational conventions $\varepsilon^{n}: G^{n} \rightarrow \mathfrak{M}$ is given the obvious definition and we also define $\delta^{n}: G \rightarrow G^{n+1}$ as any composite of $\delta$ 's. The "coassociative" law guarantees that they are all equal.

This work was partially supported by NSF grant GP-5478

(c) Michael Barr, 1969. Permission to copy for private use granted. 
Proposition 1.2. For any integers $n, m \geq 0$,

$$
\begin{array}{cl}
\varepsilon^{n} \cdot G^{i} \varepsilon^{m} G^{n-i}=\varepsilon^{n+m}, & \text { for } 0 \leq i \leq n, \\
G^{i} \delta^{m} G^{n-i} \cdot \delta^{n}=\delta^{m+n}, & \text { for } 0 \leq i \leq n \\
G^{n-i+1} \varepsilon^{m} G^{i} \cdot \delta^{n+m}=\delta^{n}, & \text { for } 0 \leq i \leq n+1, \\
\varepsilon^{n+m} \cdot G^{i} \delta G^{n-i-1}=\varepsilon^{n}, & \text { for } 0 \leq i \leq n-1 .
\end{array}
$$

The proof is given in the Appendix (A.1).

Definition 1.3. A simplicial $\mathfrak{M}$ object $X=\left\{X_{n}, d_{n}^{i} X, s_{n}^{i} X\right\}$ consists of objects $X_{n}$, $n \geq 0$, of $\mathfrak{M}$ together with morphisms $d^{i}=d_{n}^{i} X: X_{n} \rightarrow X_{n-1}$ for $0 \leq i \leq n$ called face operators and morphisms $s^{i}=s_{n}^{i} X: X_{n} \rightarrow X_{n+1}$ for $0 \leq i \leq n$ called degeneracies subject to the usual commutation identities (see, for example [Huber (1961)]). A morphism $\alpha: X \rightarrow Y$ of simplicial objects consists of a sequence $\alpha_{n}: X_{n} \rightarrow Y_{n}$ of morphisms commuting in the obvious way with all faces and degeneracies. A homotopy $h: \alpha \sim \beta$ of such morphisms consists of morphisms $h^{i}=h_{n}^{i}: X_{n} \rightarrow Y_{n+1}$ for $0 \leq i \leq n$ for each $n \geq 0$ satisfying $d^{0} h_{n}^{0}=\alpha_{n}, d^{n+1} h_{n}^{n}=\beta_{n}$ and five additional identities tabulated in [Huber (1961)].

From now on we will imagine $\mathfrak{M}$ embedded in $\mathfrak{N}$ as the subcategory of constant simplicial objects, those $X=\left\{X_{n}, d_{n}^{i}, s_{n}^{i}\right\}$ for which $X_{n}=C, d_{n}^{i}=s_{n}^{i}=C$ for all $n$ and all $0 \leq i \leq n$.

Definition 1.4. Given a cotriple $\mathbf{G}=(G, \varepsilon, \delta)$ on $\mathfrak{M}$ we define a functor $G^{*}: \mathfrak{N} \rightarrow \mathfrak{N}$ by letting $X=\left\{X_{n}, d_{n}^{i} X, s_{n}^{i} X\right\}$ and $G^{*} X=Y=\left\{Y_{n}, d_{n}^{i} Y, s_{n}^{i} Y\right\}$, where $Y_{n}=G^{n+1} X_{n}$, $d_{n}^{i} Y=G^{i} \varepsilon G^{n-i}\left(d_{n}^{i} X\right)$ and $s_{n}^{i} Y=G^{i} \delta G^{n-i}\left(s_{n}^{i} X\right) .^{\mathrm{a}}$

TheOREm 1.5. If $h: \alpha \sim \beta$ where $\alpha, \beta: X \rightarrow Y$, then $G^{*} h: G^{*} \alpha \sim G^{*} \beta$ where $\left(G^{*} h\right)_{n}^{i}=$ $G^{i} \delta G^{n-i} h_{n}^{i}$.

The proof is given in the Appendix (A.2).

THEOREM 1.6. Suppose $\mathfrak{R}$ is any subcategory of $\mathfrak{M}$ containing all the terms and all the faces and degeneracies of an object $X$ of $\mathfrak{M}$. Suppose there is a natural transformation $\vartheta: \Re \rightarrow G \mid \Re$ such that $\varepsilon \cdot \vartheta=\mathfrak{R}$. Then there are maps $\alpha: G^{*} X \rightarrow X$ and $\beta: X \rightarrow G^{*} X$ such that $\alpha \cdot \beta=X$ and $G^{*} X \sim \beta \cdot \alpha$.

The proof is given in the Appendix (A.3).

${ }^{a}$ Editor's footnote: This definition makes no sense. The definition of $d_{i}^{n}$ should be $G^{i} \varepsilon G^{n-i} \cdot G^{n+1} d_{n}^{i} X$ and similarly I should have had $s_{n}^{i} Y=G^{i} \delta G^{n-i} \cdot G^{n+1} s_{n}^{i} X$. I (the editor) no longer know what I (the author) was thinking when I wrote this. Many thanks to Don Van Osdol, who was evidently doing a lot more than proofreading, for noting this. This notation appears later in this paper too and I have decided to keep it as in the original. 
2. The distributive law

The definitions 2.1 and Theorem 2.2 were first discovered by H. Appelgate and J. Beck (unpublished).

Definition 2.1. Given cotriples $\mathbf{G}_{1}=\left(G_{1}, \varepsilon_{1}, \delta_{1}\right)$ and $\mathbf{G}_{2}=\left(G_{2}, \varepsilon_{2}, \delta_{2}\right)$ on $\mathfrak{M}$, a natural transformation $\lambda: G_{1} G_{2} \rightarrow G_{2} G_{1}$ is called a distributive law of $\mathbf{G}_{1}$ over $\mathbf{G}_{2}$ provided the following diagrams commute
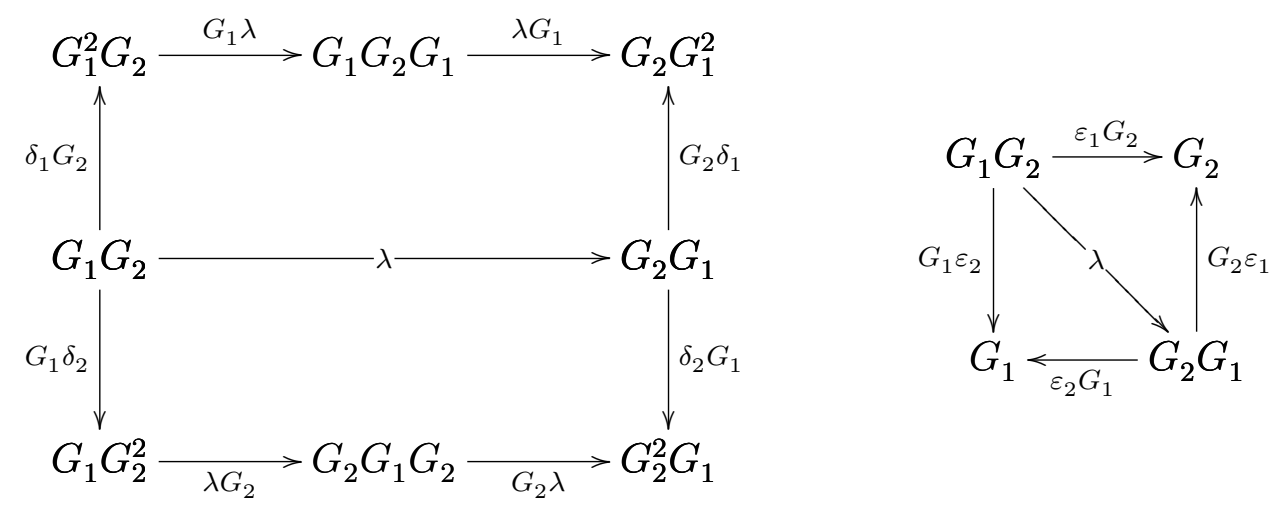

TheOREM 2.2. Suppose $\lambda: G_{1} G_{2} \rightarrow G_{2} G_{1}$ is a distributive law of $\mathbf{G}_{1}$ over $\mathbf{G}_{2}$. Let $G=G_{1} G_{2}, \varepsilon=\varepsilon_{1} \varepsilon_{2}$ and $\delta=G_{1} \lambda G_{2} \cdot \delta_{1} \delta_{2}$. Then $\mathbf{G}=(G, \varepsilon, \delta)$ is a cotriple. We write $\mathbf{G}=\mathbf{G}_{1}{ }^{\circ} \mathbf{G}_{2}$.

The proof is given in the Appendix (A.4).

Definition 2.3. For $n \geq 0$ we define $\lambda^{n}: G_{1}^{n} G_{2} \rightarrow G_{2} G_{1}^{n}$ by $\lambda^{0}=G_{2}$ and $\lambda^{n}=\lambda^{n-1} G_{1}$. $G_{1}^{n-1} \lambda$. Also $\lambda_{n}: G_{1}^{n+1} G_{2}^{n+1} \rightarrow G^{n+1}$ is defined by $\lambda_{0}=G$ and $\lambda_{n}=G_{1} G_{2} \lambda_{n-1} \cdot G_{1} \lambda^{n} G_{2}^{n}$. Let $\lambda^{*}: G_{1}^{*} G_{2}^{*} \rightarrow G^{*}$ be the natural transformation whose $n$-th component is $\lambda_{n}$.

Proposition 2.4.

(1) $G_{2}^{n} \varepsilon_{1} \cdot \lambda^{n}=\varepsilon_{1} G_{2}^{n}$,

for $n \geq 0$,

$G_{2}^{n} \delta_{1} \cdot \lambda^{n}=\lambda^{n} G_{1} \cdot G_{1} \lambda^{n} \cdot \delta G_{2}^{n}$

for $n \geq 0$,

(4)

$G_{2}^{i} \varepsilon_{2} G_{2}^{n-i} G_{1} \cdot \lambda^{n+1}=\lambda^{n} \cdot G_{1} G_{2}^{i} \varepsilon_{2} G_{2}^{n-i}, \quad$ for $0 \leq i \leq n$,

The proof is given in the Appendix (A.5).

\section{Derived Functors}

Definition 3.1. Given a functor $E: \mathfrak{M} \rightarrow \mathfrak{A}$ we define $E_{C}: \mathfrak{N} \rightarrow \mathfrak{B}$ by letting $E_{C} X$ where $X=\left\{X_{n}, d_{n}^{i}, s_{n}^{i}\right\}$ be the complex with $E X_{n}$ in degree $n$ and boundary

$$
\sum_{i=0}^{n}(-1)^{i} E d_{n}^{i}: E X_{n} \rightarrow E X_{n-1}
$$


The following proposition is well known and its proof is left to the reader.

Proposition 3.2. If $\alpha, \beta: X \rightarrow Y$ are morphisms in $\mathfrak{N}$ and $h: \alpha \sim \beta$ and we let $E_{C} h: E_{C} X_{n} \rightarrow E_{C} Y_{n+1}$ be $\sum_{i=0}^{n}(-1)^{i} E h_{n}^{i}$ then $E_{C} h: E_{C} \alpha \sim E_{C} \beta$.

Definition 3.3. If $E: \mathfrak{M} \rightarrow \mathfrak{A}$ is given, the derived functors of $E$ with respect to the cotriple $\mathbf{G}$, denoted by $\mathbf{H}(\mathbf{G} ;-, E)$, are the homology groups of the chain complex $E_{C} G^{*} X$ (where $X$ is thought of as a constant simplicial object).

Theorem 3.4. If $\mathbf{G}=\mathbf{G}_{1}{ }^{\circ}{ }_{\lambda} \mathbf{G}_{2}$ then for any $E: \mathfrak{M} \rightarrow \mathfrak{A}, E_{C} \lambda^{*}: E_{C} G_{1}^{*} G_{2}^{*} \rightarrow E_{C} G^{*}$ is a chain equivalence.

Proof. The proof uses the method of acyclic models described (in dual form) in [Barr \& Beck (1966)]. We let $V$ and $W$ be the chain complexes $E_{C} G_{1}^{*} G_{2}^{*}$ and $E_{C} G^{*}$, respectively. Then we show that $E_{C} \lambda^{*}$ induces an isomorphism of 0-homology, that both $V_{n}$ and $W_{n}$ are $\mathbf{G}$-retracts (in the sense given below- we use this term in place of $\mathbf{G}$-representable to avoid conflict with the more common use of that term) and that each becomes naturally contractible when composed with $\mathbf{G}$. For $W$, being the $\mathbf{G}$-chain complex, these properties are automatic (see [Barr \& Beck (1966)]).

Proposition 3.5. $E_{C} \lambda^{*}$ induces an isomorphism of 0-homology.

Proof. Consider the commutative diagram with exact rows

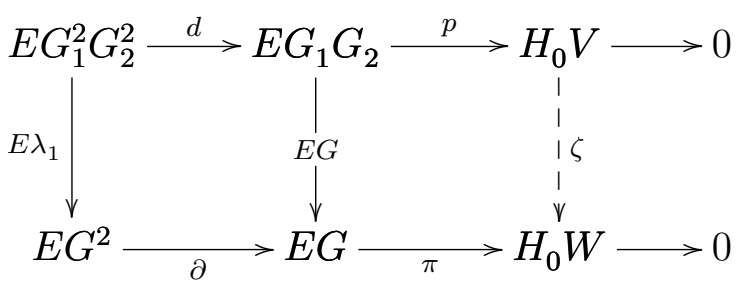

where $d=E \varepsilon_{1} G_{1} \varepsilon_{2} G_{2}-E G_{1} \varepsilon_{1} G_{2} \varepsilon_{2}, \partial=E \varepsilon G-E G \varepsilon, p=$ coker $d, \pi=$ coker $\partial$ and $\zeta$ is induced by $E G: E G_{1} G_{2} \rightarrow E G$ since $\pi \cdot d=\pi \cdot \partial \cdot E \lambda_{1}=0$. To show $\zeta$ is an isomorphism we first show that $p \cdot \partial=0$. In fact, $p \cdot E \varepsilon G=p \cdot E \varepsilon_{1} \varepsilon_{2} G_{1} G_{2}=p \cdot E \varepsilon_{1} G_{1} G_{2} \cdot E G_{1} \varepsilon_{2} G_{1} G_{2}=$ $p \cdot E \varepsilon_{1} G_{1} \varepsilon_{2} G_{2} . E G_{1} \varepsilon_{2} G_{1} \delta_{2}=p \cdot E G_{1} \varepsilon_{1} G_{2} \varepsilon_{2} \cdot E G_{1} \varepsilon_{2} G_{1} \delta_{2}=p \cdot E G_{1} \varepsilon_{1} \varepsilon_{2} G_{2}$. In a similar way this is also equal to $p \cdot E G \varepsilon$ and so $p \cdot \partial=0$. But then there is a $\xi: H_{0} W \rightarrow H_{0} V$ such that $\xi \cdot \pi=p$. But then $\xi \cdot \zeta \cdot p=\xi \cdot \pi=p$ from which, since $p$ is an epimorphism we conclude $\xi \cdot \zeta=H_{0} V$. Similarly $\zeta \cdot \xi=H_{0} W$.

Now we return to the proof of 3.4. To say that $V_{n}$ is a $\mathbf{G}$-retract means that there are maps $\vartheta_{n}: V_{n} \rightarrow V_{n} G$ such that $V_{n} \varepsilon \cdot \vartheta_{n}=V_{n}$. Let $\vartheta_{n}=E_{C} G_{1}^{n}\left(G_{1} \lambda^{n+1} G_{2} \cdot \delta_{1} G_{2}^{n} \delta_{2}\right)$. Then $V_{n} \varepsilon \cdot \vartheta_{n}=E_{C} G_{1}^{n+1} G_{2}^{n+1} \varepsilon_{1} \varepsilon_{2} \cdot E_{C} G_{1}^{n}\left(G_{1} \lambda^{n+1} G_{2} \cdot \delta_{1} G_{2}^{n} \delta_{2}\right)=E_{C} G_{1}^{n}\left(G_{1} G_{2}^{n+1} \varepsilon_{1} \varepsilon_{2} \cdot G_{1} \lambda^{n+1} G_{2} \cdot\right.$ $\left.\delta_{1} G_{2}^{n} \delta_{2}\right)=E_{C} G_{1}^{n}\left(G_{1} \varepsilon_{1} G_{2}^{n+1} \cdot \delta_{1} G_{2}^{n} \delta_{2}\right)=E G_{1}^{n}\left(G_{1} G_{2}^{n+1}\right)=V_{n}$.

To see that the augmented complex $V G \rightarrow H_{0} V G \rightarrow 0$ has a natural contracting homotopy, observe that for any $X$ the constant simplicial object $G X$ satisfies Theorem 1.6 with respect to the cotriples $\mathbf{G}_{1}$ and $\mathbf{G}_{2}$, taking $\mathfrak{R}$ to be the full subcategory generated 
by the image of $G$. In fact $\delta_{1} G_{2} X: G X \rightarrow G_{1} G X$ and $\lambda G_{2} X \cdot G_{1} \delta_{2} X: G X \rightarrow G_{2} G X$ are natural maps whose composite with $\varepsilon_{1} G X$ and $\varepsilon_{2} G X$, respectively, is the identity. This means, for $i=1,2$, that the natural map $\alpha_{i} X: G_{i}^{*} G X \rightarrow G X$ whose $n$-th component is $\varepsilon_{i}^{n+1} G X$ has a homotopy inverse $\beta_{i} X: G X \rightarrow G_{i}^{*} G X$ with $\alpha_{i} \cdot \beta_{i}=G$. Let $h_{i}: G_{i}^{*} G \sim \beta_{i} \cdot \alpha_{i}$ denote the natural homotopy. Then if $\alpha=\alpha_{1} \cdot G_{1}^{*} \alpha_{2}, \beta=G_{1}^{*} \beta_{2} \cdot \beta_{1}$ we have $E_{c} \alpha: E_{C} G_{1}^{*} G_{2}^{*} G \rightarrow E_{C} G$ and $E_{C} \beta: E_{C} G \rightarrow E_{C} G_{1}^{*} G_{2}^{*} G$. Moreover, noting that the boundary operator in $E_{C} G$ simply alternates 0 and $E G$ it is obvious that the identity map of degree 1 denoted by $h_{3}$ is a contracting homotopy. Then if

$$
\begin{aligned}
h= & E_{C} G_{1}^{*} h_{2}+E_{C}\left(G_{1}^{*} \beta_{2} \cdot h_{1} \cdot G_{1}^{*} \alpha_{2}\right)+E_{C}\left(\beta \cdot h_{3} \cdot \alpha\right), \\
d \cdot h+h \cdot d= & d \cdot E_{C} G_{1}^{*} h_{2}+d \cdot E_{C}\left(G_{1}^{*} \beta_{2} \cdot h_{1} \cdot G_{1}^{*} \alpha_{2}\right)+d \cdot E_{C}\left(\beta \cdot h_{3} \cdot \alpha\right)+E_{C} G_{1}^{*} h_{2} \cdot d \\
& +E_{C}\left(G_{1}^{*} \beta_{2} \cdot h_{1} \cdot G_{1}^{*} \alpha_{2}\right) \cdot d+E_{C}\left(\beta \cdot h_{3} \cdot \alpha\right) \cdot d \\
= & E_{C}\left(G_{1}^{*} G_{2}^{*} G-G_{1}^{*}\left(\beta_{2} \cdot \alpha_{2}\right)\right)+E_{C} G_{1}^{*} \beta_{2} \cdot E_{C}\left(d h_{1}+h_{1} d\right) \cdot E_{C} G_{1}^{*} \alpha_{2} \\
& +E_{C} \beta \cdot E_{C}\left(d h_{3}+h_{3} d\right) \cdot E_{C} \alpha \\
= & V G-E_{C} G_{1}^{*}\left(\beta_{2} \cdot \alpha_{2}\right)+E_{C} G_{1}^{*} \beta_{2} \cdot E_{C}\left(G_{1}^{*} G-\beta_{1} \cdot \alpha_{1}\right) \cdot E_{C} G_{1}^{*} \alpha_{2} \\
& +E_{C}(\beta \cdot \alpha) \\
= & V G-E_{C} G_{1}^{*}\left(\beta_{2} \cdot \alpha_{2}\right)+E_{C} G_{1}^{*}\left(\beta_{2} \cdot \alpha_{2}\right)-E_{C}\left(G_{1}^{*} \beta_{2} \cdot \beta_{1} \cdot \alpha_{1} \cdot G_{1}^{*} \alpha_{2}\right) \\
& +E_{C}(\beta \cdot \alpha) \\
= & V G .
\end{aligned}
$$

This completes the proof.

\section{Simplicial Algebras}

In this section we generalize from the category of associative $k$-algebras to the category of simplicial associative $k$-algebras the theorem of [Barr \& Beck (1966)] which states that the triple cohomology with respect to the underlying category of $k$-modules is equivalent to a "suspension" of the Hochschild cohomology. The theorem we prove will be easily seen to reduce to the usual one for a constant simplicial object.

Let $\Lambda$ be an ordinary algebra. We let $\mathfrak{M}$ be the category of $k$-algebras over $\Lambda$. More precisely, an object of $\mathfrak{M}$ is a $\Gamma \rightarrow \Lambda$ and a morphism of $\mathfrak{M}$ is a commutative triangle $\Lambda \leftarrow \Gamma \rightarrow \Gamma^{\prime} \rightarrow \Lambda$. In what follows we will normally drop any explicit reference to $\Lambda$. As before we let $\mathfrak{N}$ denote the category of simplicial $\mathfrak{M}$ objects. Let $\mathbf{G}_{t}$ denote the tensor algebra cotriple on $\mathfrak{M}$ lifted to $\mathfrak{N}$ in the obvious way: $G_{t}\left\{X_{n}, d^{i}, s^{i}\right\}=\left\{G_{t} X_{n}, G_{t} d^{i}, G_{t} s^{i}\right\}$. Let $G_{p}$ denote the functor on $\mathfrak{N}$ described by $G_{p}\left\{X_{n}, d_{n}^{i}, s_{n}^{i}\right\}=\left\{X_{n+1}, d_{n+1}^{i+1}, s_{n+1}^{i+1}\right\}$. This means that the $n$-th term is $X_{n+1}$ and the $i$-th face and degeneracy are $d^{i+1}$ and $s^{i+1}$ respectively. Let $\varepsilon_{p}: G_{p} X \rightarrow X$ be the map whose $n$-th component is $d_{n+1}^{0}$ and $\delta_{p}: G_{p} X \rightarrow G_{p}^{2} X$ be the map whose $n$-th component is $s_{n+1}^{0}$.

Proposition 4.1.

(1) $\mathbf{G}_{p}=\left(G_{p}, \varepsilon_{p}, \delta_{p}\right)$ is a cotriple; in particular $\varepsilon_{p}$ and $\delta_{p}$ are simplicial maps. 
(2) If $\mathbf{G}$ is any cotriple "lifted" from a cotriple on $\mathfrak{M}$, then the equality $G G_{p}=G_{p} G$ is a distributive law.

(3) The natural transformations $\alpha$ and $\beta$ where $\alpha X: G_{p} X \rightarrow X_{0}$ whose $n$-th component is $d^{1} \cdot d^{1} \cdots \cdot d^{1}$ and $\beta X: X_{0} \rightarrow G_{p} X$ whose $n$-th component is $s^{0} \cdot s^{0} \cdots \cdot s^{0}$ are maps between $G_{p} X$ and the constant object $X_{0}$ such that $\alpha \cdot \beta=X_{0}$. There is a natural homotopy $h: G_{p} X \sim \beta \cdot \alpha$.

Proof. (1) The simplicial identity $d^{0} d^{i+1}=d^{i} d^{0}, i>0$, says that $d^{0}$ commutes with the face maps. The identity $d^{0} s^{i+1}=s^{i} d^{0}, i>0$, does the same for the degeneracies and so $\varepsilon_{p}$ is simplicial. For $\delta_{p}$ we have $s^{0} d^{i+1}=d^{i+2} s^{0}$ and $s^{0} s^{i+1}=s^{i+2} s^{0}$ for $i>0$, so it is simplicial. $G_{p} \delta_{p}$ has $n$-th component $s_{n+2}^{0}$ and $\delta_{p} G_{p}$ has $n$-th component $s_{n+2}^{1}$, and so $\delta_{p} G_{p} \cdot \delta_{p}=s_{n+2}^{1} \cdot s_{n+1}^{0}=s_{n+2}^{0} \cdot s_{n+1}^{0}=G_{p} \delta_{p} \cdot \delta_{p}$, which is the coassociative law. Finally, $\varepsilon_{p} G_{p} \cdot \delta_{p}=d_{n+2}^{1} \cdot s_{n+1}^{0}=X_{n+1}=d_{n+2}^{0} \cdot s_{n+1}^{0}=G_{p} \varepsilon_{p} \cdot \delta_{p}$.

(2) This is completely trivial.

(3) This is proved in the Appendix (A.6).

We note that under the equivalence between simplicial sets and simplicial topological spaces the "same" functor $G_{p}$ is analogous to the topological path space.

From this we have the cotriple $\mathbf{G}=\mathbf{G}_{t} \circ \mathbf{G}_{p}$ where the distributive law is the identity map. If we take as functor the contravariant functor $E$, whose value at $X$ is $\operatorname{Der}\left(\pi_{0} X, M\right)$ where $M$ is a $\Lambda$-bimodule, the $\mathbf{G}$-derived functors are given by the homology of the cochain complex $0 \rightarrow \operatorname{Der}\left(\pi_{0} G X, M\right) \rightarrow \cdots \rightarrow \operatorname{Der}\left(\pi_{0} G^{n+1} X, M\right) \rightarrow \cdots . \pi_{0} X$ is most easily described as the coequalizer of $X_{1} \rightrightarrows X_{0}$. Let $d^{0}=d_{0}^{0}: X_{0} \rightarrow \pi_{0} X$ be the coequalizer map. But by the above, $\pi_{0} G X \bumpeq G_{t} X_{0}$ and $G_{t} X=\varepsilon_{t} d^{0}$. Then $\pi_{0} G^{n+1} X=G_{t}^{n+1} X_{n}$ and the $i$-th face is $G_{t}^{i} \varepsilon_{t} G_{t}^{n-i} d^{i}$. Thus $\mathbf{H}(\mathbf{G} ; X, E)$ is just the homology of $K X$, the cochain complex whose $n$-th term is $\operatorname{Der}\left(G_{t}^{n+1} X_{n}, M\right)$. When $X$ is the constant object $\Gamma$, this reduces to the cotriple cohomology of $\Gamma$ with respect to $\mathbf{G}_{t}$.

If $X$ is in $\mathfrak{N}$, the normalized chain complex $N X$ given by $N_{n} X=\bigcap_{i=1}^{n} \operatorname{ker} d_{n}^{i b}$ naturally bears the structure of a $D G$-algebra. In fact, if $N X \otimes N X$ is the tensor product in the category of $D G$ modules over $k$ given by $(N X \otimes N X)_{n}=\sum N_{i} X \otimes N_{n-i} X$ and $X \otimes X$ is the tensor product in the category of simplicial $k$-modules given by $(X \otimes X)_{n}=$ $X_{n} \otimes X_{n}$, then the Eilenberg-Zilber map $g: N X \otimes N X \rightarrow N(X \otimes X)$ is known to be associative in the sense that $g \cdot(N X \otimes g)=g \cdot(g \otimes N X)$. From this it follows easily that if $\mu: X \otimes X \rightarrow X$ is the multiplication map in $X$, then $N \mu \cdot g$ makes $N X$ into a $D G$-algebra. Actually it can be shown that the Dold-Puppe equivalence ([Dold \& Puppe (1961)]) between the categories of simplicial $k$-modules and $D G$-modules (chain complexes) induces an analogous equivalence between the categories of simplicial algebras and $D G$-algebras. Given a $D G$-algebra $V \stackrel{\alpha}{\longrightarrow} \Lambda$, we let $\widetilde{B} V$ be the chain complex given by $\widetilde{B}_{n} V=\sum \Lambda \otimes V_{i_{1}} \otimes \cdots \otimes V_{i_{m}} \otimes \Lambda$, the sum taken over all sets of indices for which $i_{1}+\cdots+i_{m}+m=n$. The boundary $\partial=\partial \widetilde{B}$ is given by $\partial=\partial^{\prime}+\partial^{\prime \prime}$ where $\partial^{\prime}$ is the

\footnotetext{
${ }^{b}$ Editor's footnote: $N_{0} X=X_{0}$; an empty intersection of subobjects of an object is the object itself
} 
Hochschild boundary and $\partial^{\prime \prime}$ arises out of boundary in $V$. Let $\lambda\left[v_{1}, \ldots, v_{m}\right] \lambda^{\prime}$ denote the chain $\lambda \otimes v_{1} \otimes \cdots \otimes v_{m} \otimes \lambda^{\prime}, \operatorname{deg}\left[v_{1}, \ldots, v_{m}\right]$ denote the total degree of $\left[v_{1}, \ldots, v_{m}\right]$, and $\exp q$ denote $(-1)^{q}$ for an integer $q$. Then

$$
\begin{aligned}
\partial^{\prime}\left[v_{1}, \ldots, v_{m}\right] & =\alpha\left(v_{1}\right)\left[v_{2}, \ldots, v_{m}\right]+\sum \exp \left(\operatorname{deg}\left[v_{1}, \ldots, v_{i}\right]\right)\left[v_{1}, \ldots, v_{i} v_{i+1}, \ldots, v_{m}\right] \\
& +\exp \left(\operatorname{deg}\left[v_{1}, \ldots, v_{n-1}\right]\right)\left[v_{1}, \ldots, v_{n-1}\right] \alpha\left(v_{n}\right) \\
\partial^{\prime \prime}\left[v_{1}, \ldots, v_{m}\right] & =\sum \exp \left(\operatorname{deg}\left[v_{1}, \ldots, v_{i-1}\right]\right)\left[v_{1}, \ldots, d v_{i}, \ldots, v_{m}\right]
\end{aligned}
$$

where $d$ is the boundary in $V$. Then it may easily be seen that $\partial^{\prime} \partial^{\prime \prime}+\partial^{\prime \prime} \partial^{\prime}=0$, and so $\partial \widetilde{B}=\partial^{\prime}+\partial^{\prime \prime}$ is a boundary operator. It is clear that $\widetilde{B}$ reduces to the usual Hochschild complex when $V$ is concentrated in degree zero.

$B V$ is defined by letting $B_{n} V=\widetilde{B}_{n+1} V$ and $\partial B=-\partial \widetilde{B}$. This is where the degree shift in the comparison theorems between triple cohomology and the classical theories comes in. Then we define for a simplicial algebra over $\Lambda$ and $M$ a $\Lambda$-bimodule

$$
L X=\operatorname{Hom}_{\Lambda-\Lambda}(B N X, M)
$$

THEOREM 4.2. The cochain complexes $K$ and $L$ are homotopy equivalent.

Proof. We apply the theorem of acyclic models of [Barr \& Beck (1966)] with respect to G. As usual, the complex $K$, being the cotriple resolution, automatically satisfies both hypotheses of that theorem. Let $\vartheta^{n}: L^{n} G \rightarrow L^{n}$ (where $L^{n}$ is the $n$-th term of $L$ ) be the map described as follows. We have for each $n \geq 0$ a $k$-linear map $\varphi_{n} X: X_{n} \rightarrow(G X)_{n}$ given by the composite $X_{n} \stackrel{s^{0}}{\longrightarrow} X_{n+1}=G_{p} X_{n} \longrightarrow\left(G_{t} G_{p} X\right)_{n}$ where the second is the isomorphism of an algebra with the terms of degree 1 in its tensor algebra. Also it is clear that $\varepsilon X \cdot \varphi_{n} X=X_{n}$. Thus we have $k$-linear maps $\widetilde{\varphi}_{n}: N_{n} \rightarrow N_{n} G$ with $N_{n} \varepsilon \cdot \widetilde{\varphi}_{n}=N_{n}$. This comes about because $N$ is defined on the level of the underlying modules and extends to algebras. Then the $\Lambda$-bilinear map

$$
\Lambda \otimes \widetilde{\varphi}_{i_{1}} \otimes \cdots \otimes \widetilde{\varphi}_{i_{m}} \otimes \Lambda: \Lambda \otimes N_{i_{1}} \otimes \cdots \otimes N_{i_{m}} \otimes \Lambda \rightarrow \Lambda \otimes N_{i_{1}} G \otimes \cdots \otimes N_{i_{m}} G \otimes \Lambda
$$

is a map whose composite with the map induced by $\varepsilon$ is the identity. Then forming the direct sum of all those maps $\left(^{*}\right)$ for which $i_{1}+i_{2}+\cdots+i_{m}+m=n+1$ we have the map of $B_{n} \rightarrow B_{n} G$ whose composite with $B_{n} \varepsilon$ is $B_{n}$. Let $\vartheta^{n}: \operatorname{Hom}_{\Lambda-\Lambda}\left(B_{n} G, M\right) \longrightarrow$ $\operatorname{Hom}_{\Lambda-\Lambda}\left(B_{n}, M\right)$ be the map induced. Clearly $\vartheta^{n} \cdot L^{n} \varepsilon=L^{n}$.

Now we wish to show that the augmented complex $L^{+} G X=L G X \leftarrow H^{0}(L G X) \leftarrow 0$ is naturally contractible. First note that by Proposition 4.1 (3) there are natural maps $\alpha=\alpha G_{t} X: G X=G_{p} G_{t} X \rightarrow G_{t} X_{0}$ and $\beta=\beta G_{t} X: G_{t} X_{0} \rightarrow G X$ with $\alpha \cdot \beta=G_{t} X_{0}$, and there is a natural homotopy $h: G X \sim \beta \cdot \alpha$. Then we have $L^{+} \alpha: L^{+} G X \rightarrow L^{+} G_{t} X_{0}$ and $L^{+} \beta: L^{+} G_{t} X_{0} \rightarrow L^{+} G X$ such that $L^{+} \alpha \cdot L^{+} \beta=L^{+} G_{t} X_{0}$ and $L^{+} h: L^{+} G X \sim L^{+} \beta \cdot L^{+} \alpha$. If we can find a contracting homotopy $t$ in $L^{+} G_{t} X_{0}$, then $s=h+L^{+} \beta \cdot t \cdot L^{+} \alpha$ will satisfy $d s+s d=d h+h d+L^{+} \beta \cdot(d t+t d) \cdot L^{+} \alpha=L^{+} G X-L^{+} \beta \cdot L^{+} \alpha+L^{+} \beta \cdot L^{+} \alpha=L^{+} G X$. But 
$N G_{t} X_{0}$ is just the normalized complex associated with a constant. For $n>0, \bigcap_{i>0} \operatorname{ker} d_{n}^{i}=$ 0 , since each $d_{n}^{i}=G_{t} X_{0}$. Thus $N G_{t} X_{0}$ is the $D G$-algebra consisting of $G_{t} X_{0}$ concentrated in degree zero. But then $L G_{t} X_{0}$ is simply the Hochschild complex with degree lowered by one. I.e. $L G_{t} X_{0}$ is the complex $\cdots \rightarrow\left(G_{t} X_{0}\right)^{(4)} \rightarrow\left(G_{t} X_{0}\right)^{(3)} \rightarrow 0$ with the usual boundary operator. But this complex was shown to be naturally contractible in [Barr (1966)]. In fact this was the proof that the Hochschild cohomology was essentially the triple cohomology with respect to $\mathbf{G}_{t}$. What remains in order to finish the proof of theorem 4.2 is to show:

Proposition 4.3. $H^{0}(K) \simeq H^{0}(L) \simeq \operatorname{Der}\left(\pi_{0} X, M\right)$.

An auxiliary proposition will be needed. It is proved in the Appendix (A.7).

Proposition 4.4. If $X$ is as above, then $\varepsilon_{t} d^{0}: G_{t} X_{0} \rightarrow \pi_{0} X$ is the coequalizer of $\varepsilon_{t} G_{t} d^{0}$ and $G_{t} \varepsilon_{t} d^{1}$ from $G_{t}^{2} X_{1}$ to $G_{t} X_{0}$.

Proof of Proposition 4.3. From Proposition 4.4 it follows that for any $\Gamma, \mathfrak{M}\left(\pi_{0} X, \Gamma\right)$ is the equalizer of $\mathfrak{M}\left(G_{t} X_{0}, \Gamma\right) \rightrightarrows \mathfrak{M}\left(G_{t}^{2} X_{1}, \Gamma\right)$. But by letting $\Gamma$ be the split extension $\Lambda \times M$ and using the well-known fact $\operatorname{Der}(Y, M) \simeq \mathfrak{M}(Y, \Lambda \times M)$ for any $Y$ of $\mathfrak{M}$, we have that $\operatorname{Der}\left(\pi_{0} X, M\right)$ is the equalizer of $\operatorname{Der}\left(G_{t} X_{0}, \Gamma\right) \rightrightarrows \operatorname{Der}\left(G_{t}^{2} X_{1}, \Gamma\right)$ or simply the kernel of the difference of the two maps. I.e. $\operatorname{Der}\left(\pi_{0} X, M\right)$ is the kernel of $K^{0} X \rightarrow K^{1} X$ and thus is isomorphic to $H^{0} K X$.

To compute $H^{0} L$, it suffices to show that $H_{0}(B N X)=$ Diff $\pi_{0} X$ where, for an algebra $\varphi: \Gamma \rightarrow \Lambda$, Diff $\Gamma$ represents $\operatorname{Der}(\Gamma,-)$ on the category of $\Lambda$-modules. Explicitly, Diff $\Gamma$ is the cokernel of $\Lambda \otimes \Gamma \otimes \Gamma \otimes \Lambda \rightarrow \Lambda \otimes \Gamma \otimes \Lambda$ where the map is the Hochschild boundary operator $\partial\left(\lambda \otimes \gamma \otimes \gamma^{\prime} \otimes \lambda^{\prime}\right)=\lambda \cdot \varphi \gamma \otimes \gamma^{\prime} \otimes \lambda^{\prime}-\lambda \otimes \gamma \gamma^{\prime} \otimes \lambda^{\prime}+\lambda \otimes \gamma \otimes \varphi \gamma^{\prime} \cdot \lambda^{\prime}$. If for convenience we denote the cokernel of an $f: A \rightarrow B$ by $B / A$, we have $\pi_{0} X=N_{0} X / N_{1} X$, and then

$$
\begin{aligned}
H_{0}(B N X) & =\frac{\Lambda \otimes N_{0} X \otimes \Lambda}{\Lambda \otimes N_{1} X \otimes \Lambda+\Lambda \otimes N_{0} X \otimes N_{0} X \otimes \Lambda} \simeq \frac{\Lambda \otimes \pi_{0} X \otimes \Lambda}{\Lambda \otimes N_{0} X \otimes N_{0} X \otimes \Lambda} \\
& \simeq \frac{\Lambda \otimes \pi_{0} X \otimes \Lambda}{\Lambda \otimes \pi_{0} X \otimes \pi_{0} X \otimes \Lambda} \simeq \operatorname{Diff} \pi_{0} X
\end{aligned}
$$

The next to last isomorphism comes from the fact that $\Lambda \otimes N_{0} X \otimes N_{0} X \otimes \Lambda \longrightarrow \Lambda \otimes \pi_{0} X \otimes \Lambda$ factors through the surjection $\Lambda \otimes N_{0} X \otimes N_{0} X \otimes \Lambda \rightarrow \Lambda \otimes \pi_{0} X \otimes \pi_{0} X \otimes \Lambda$. This argument is given by element chasing in [Barr (1967)], Proposition 3.1.

We now recover the main theorem 1.1. of [Barr (1967)] as follows.

Definition 4.5. Given a k-algebra $\Gamma \rightarrow \Lambda$ we define $G_{k} \Gamma \rightarrow \Lambda$ by letting $G_{k} \Gamma$ be the free $k$-module on the elements of $\Gamma$ made into an algebra by letting the multiplication in $\Gamma$ define the multiplication on the basis. That is, if $\gamma_{1}, \gamma_{2} \in \Gamma$ and if $\left[\gamma_{i}\right]$ denotes the basis element of $G_{k} \Gamma$ corresponding to $\gamma_{i}, i=1,2$, then $\left[\gamma_{1}\right]\left[\gamma_{2}\right]=\left[\gamma_{1} \gamma_{2}\right]$. 
THEOREM 4.6. There are natural transformations $\varepsilon_{k}$ and $\delta_{k}$ such that $\mathbf{G}_{k}=\left(G_{k}, \varepsilon_{k}, \delta_{k}\right)$ is a cotriple. Also there is a natural $\lambda: G_{t} G_{k} \rightarrow G_{k} G_{t}$ which is a distributive law.

Proof. $\varepsilon_{k}: G_{k} \Gamma \rightarrow \Gamma$ takes $[\gamma]$ to $\gamma$ and $\delta_{k}$ takes $[\gamma]$ to $[[\gamma]]$ for $\gamma \in \Gamma . G_{k}$ is made into a functor by $G_{k} f[\gamma]=[f \gamma]$ for $f: \Gamma \rightarrow \Gamma^{\prime}$ and $\gamma \in \Gamma$. Then

$$
G_{k} \delta_{k} \cdot \delta_{k}[\gamma]=G_{k} \delta_{k}[[\gamma]]=\left[\delta_{k}[\gamma]\right]=[[[\gamma]]]=\delta_{k} G_{k}[[\gamma]]=\delta_{k} G_{k} \cdot \delta_{k}[\gamma]
$$

Also

$$
G_{k} \varepsilon_{k} \cdot \delta_{k}[\gamma]=G_{k} \varepsilon_{k}[[\gamma]]=\left[\varepsilon_{k}[\gamma]\right]=[\gamma]=\varepsilon_{k} G_{k}[[\gamma]]=\varepsilon_{k} G_{k} \cdot \delta_{k}[\gamma]
$$

To define $\lambda$ we note that $G_{t} G_{k} \Gamma$ is the free algebra on the set underlying $\Gamma$. In fact, any algebra homomorphism $G_{t} G_{k} \Gamma \rightarrow \Gamma^{\prime}$ is, by adjointness of the tensor product with the underlying $k$-module functor, determined by its value on the $k$-module underlying $G_{k} \Gamma$. As a $k$-module this is simply free on the set underlying $\Gamma$. Thus an algebra homomorphism $G_{t} G_{k} \Gamma \rightarrow G_{k} G_{t} \Gamma$ is prescribed by a set map of $\Gamma \rightarrow G_{k} G_{t} \Gamma$. Let $\langle\gamma\rangle$ denote the element of $G_{t} \Lambda$ corresponding to $\gamma \in \Gamma$. Then $\lambda\langle[\gamma]\rangle=[\langle\gamma\rangle]$ is the required map. In this form the laws that must be verified become

completely transparent. For example,

$$
\begin{aligned}
\lambda G_{t} \cdot G_{t} \lambda \cdot \delta_{t} G_{k}\langle[\gamma]\rangle & =\lambda G_{t} \cdot G_{t} \lambda\langle\langle[\gamma]\rangle\rangle=\lambda G_{t} \cdot\langle\lambda\langle[\gamma]\rangle\rangle=\lambda G_{t}\langle[\langle\gamma\rangle]\rangle \\
& =[\langle\langle\gamma\rangle\rangle]=\left[\delta_{t}\langle\gamma\rangle\right] G_{k} \delta_{t}[\langle\gamma\rangle]=G_{k} \delta_{t} \cdot \lambda\langle[\gamma]\rangle
\end{aligned}
$$

The remaining identities are just as easy. It is, however, instructive to discuss somewhat more explicitly what $\lambda$ does to a more general element of $G_{t} G_{k} \Gamma$.

A general element of $G_{t} G_{k} \Gamma$ is a formal (tensor) product of elements which are formal $k$-linear combinations of elements of $\Gamma$. We are required to produce from this an element of $G_{k} G_{t} \Gamma$ which is a formal $k$-linear combination of formal products of elements of $\Gamma$. Clearly the ordinary distributive law is exactly that: a prescription for turning a product of sums into a sum of products. For example $\lambda\left(\langle[\gamma]\rangle \otimes\left(\left\langle\alpha_{1}\left[\gamma_{1}\right]+\cdots+\alpha_{n}\left[\gamma_{n}\right]\right\rangle\right)\right)=\alpha_{1}\left[\langle\gamma\rangle \otimes\left\langle\gamma_{1}\right\rangle\right]+$ $\cdots+\alpha_{n}\left[\langle\gamma\rangle \otimes\left\langle\gamma_{n}\right\rangle\right]$. The general form is practically impossible to write down but the idea should be clear. It is from this example that the term "distributive law" comes.

Now $G_{k}^{*} \Gamma$ is, for any $\Gamma \rightarrow \Lambda$, an object of $\mathfrak{N}$. Its cohomology with respect to $G=G_{p} G_{t}$ is with coefficients in the $\Lambda$-module $M$, as we have seen, the cohomology of $0 \rightarrow \operatorname{Der}\left(G_{t} G_{k} \Gamma, M\right) \rightarrow \cdots \rightarrow \operatorname{Der}\left(G_{t}^{n+1} G_{k}^{n+1} \Gamma, M\right) \rightarrow \cdots$ which by theorem 3.4 is chain equivalent to $0 \rightarrow \operatorname{Der}\left(G_{t} G_{k} \Gamma, M\right) \rightarrow \cdots \operatorname{Der}\left(\left(G_{t} G_{k}\right)^{n+1} \Gamma, M\right) \rightarrow \cdots$, in other words the cohomology of $\Gamma$ with respect to the free algebra cotriple $G_{t} G_{k}$. On the other hand, $N G_{k} \Gamma$ is a $D G$-algebra, acyclic and $k$-projective in each degree. Thus $B N G_{k} \Gamma$ is, except for the dimension shift, exactly Shukla's complex. Thus if $\operatorname{Shuk}^{n}(\Gamma, M)$ denotes the Shukla cohomology groups as given in [Shukla (1961)], the above, together with Proposition 4.3 shows:

THEOREM 4.7. There are natural isomorphisms

$$
H^{n}\left(\mathbf{G}_{t}{ }_{\lambda} \mathbf{G}_{k} ; \Gamma, M\right) \simeq \begin{cases}\operatorname{Der}(\Gamma, M), & n=0 \\ \operatorname{Shuk}^{n+1}(\Gamma, M), & n>0\end{cases}
$$




\section{Other applications}

In this section we apply the theory to get two theorems about derived functors, each previously known in cohomology on other grounds.

TheOREM 5.1. Let $\mathbf{G}_{f}$ and $\mathbf{G}_{b f}$ denote the cotriples on the category of groups for which $G_{f} X$ is the free group on the elements of $X$ and $G_{b f} X$ is the free group on the elements of $X$ different from 1. ${ }^{\mathrm{c}}$ Then the $\mathbf{G}_{f}$ and $\mathbf{G}_{b f}$ derived functors are equivalent.

THEOREM 5.2. Let $\mathfrak{M}$ be the category of $k$-algebras whose underlying $k$-modules are $k$ projective. Then if $\mathbf{G}_{t}, \mathbf{G}_{k}$ and $\lambda$ are as above (Section 4 ), the $\mathbf{G}_{t}$ and $\mathbf{G}_{t}{ }^{\circ} \mathbf{G}_{k}$ derived functors are equivalent.

Before beginning the proofs we need the following:

Definition 5.3. If $\mathbf{G}$ is a cotriple on $\mathfrak{M}$, then an object $X$ of $\mathfrak{M}$ is said to be $\mathbf{G}$-projective if there is a sequence $X \stackrel{\alpha}{\longrightarrow} G Y \stackrel{\beta}{\longrightarrow} X$ with $\beta \cdot \alpha=X$. We let $P(\mathbf{G})$ denote the class of all $\mathbf{G}$-projectives.

The following theorem is shown in [Barr \& Beck (1969)].

TheOREM 5.4. If $\mathbf{G}_{1}$ and $\mathbf{G}_{2}$ are cotriples on $\mathfrak{M}$ with $P\left(\mathbf{G}_{1}\right)=P\left(\mathbf{G}_{2}\right)$, then the $\mathbf{G}_{1}$ and $\mathbf{G}_{2}$ derived functors are naturally equivalent.

Proposition 5.5. Suppose $\mathbf{G}_{1}$ and $\mathbf{G}_{2}$ are cotriples on $\mathfrak{M}, \lambda: G_{1} G_{2} \rightarrow G_{2} G_{1}$ is a distributive law, and $\mathbf{G}=\mathbf{G}_{1}{ }^{\circ} \mathbf{G}_{2}$. Then $P(\mathbf{G})=P\left(\mathbf{G}_{1}\right) \cap P\left(\mathbf{G}_{2}\right)$.

Proof. If $X$ is $\mathbf{G}$-projective, it is clearly $\mathbf{G}_{1}$-projective. If $X \stackrel{\alpha}{\longrightarrow} G_{1} G_{2} Y \stackrel{\beta}{\longrightarrow} X$ is a sequence with $\beta \cdot \alpha=X$, then

$$
X \stackrel{\alpha}{\longrightarrow} G_{1} G_{2} Y \stackrel{G_{1} \delta_{2}}{\longrightarrow} G_{1} G_{2}^{2} Y \stackrel{\lambda G_{2} Y}{\longrightarrow} G_{2} G_{1} G_{2} Y \stackrel{\varepsilon_{2} \beta}{\longrightarrow} X
$$

is a sequence whose composite is $X$. If $X$ is both $\mathbf{G}_{1}$ - and $\mathbf{G}_{2}$-projective, find

$$
X \stackrel{\alpha_{i}}{\longrightarrow} G_{i} Y_{i} \stackrel{\beta_{i}}{\longrightarrow} X
$$

for $i=1,2$, with $\beta_{i} \cdot \alpha_{i}=X$; then

$$
X \stackrel{\alpha_{1}}{\longrightarrow} G_{1} Y_{1} \stackrel{\delta_{1} Y_{1}}{\longrightarrow} G_{1}^{2} Y_{1} \stackrel{G_{1} \beta_{1}}{\longrightarrow} G_{1} X \stackrel{G_{1} \alpha_{2}}{\longrightarrow} G_{1} G_{2} Y_{2} \stackrel{\varepsilon_{1} G_{2} Y_{2}}{\longrightarrow} G_{2} Y_{2} \stackrel{\beta_{2}}{\longrightarrow} X
$$

\footnotetext{
${ }^{c}$ Editor's footnote: On first glance, it is not obvious why $G_{b f}$ is even a functor, let alone a cotriple. We leave it an exercise for the reader to show that $\mathbf{G}_{b f}$ can be factored by an adjunction as follows. Let $\mathbf{P F}$ denote the category of sets and partial functions. Let $U_{b f}$ : Groups $\longrightarrow \mathbf{P F}$ that takes a group to the elements different from the identity, while $F_{b f}: \mathbf{P F} \longrightarrow$ Groups takes a set to the free group generated by it and when $f: X \longrightarrow Y$ is a partial function, $F_{b f} f$ takes every element not in dom $f$ to the identity.
} 
is a sequence for which

$$
\begin{array}{r}
\beta_{2} \cdot \varepsilon_{1} G_{2} Y \cdot G_{1} \alpha_{2} \cdot G_{1} \beta_{1} \cdot \delta_{1} Y_{1} \cdot \alpha_{1}=\varepsilon_{1} X \cdot G_{1} \beta_{2} \cdot G_{1} \alpha_{2} \cdot G_{1} \beta_{1} \cdot \delta_{1} Y_{1} \cdot \alpha_{1} \\
=\varepsilon_{1} X \cdot G_{1} \beta_{1} \cdot \delta_{1} Y_{1} \cdot \alpha_{1}=\beta_{1} \cdot \varepsilon_{1} G_{1} Y_{1} \cdot \delta_{1} Y_{1} \cdot \alpha_{1}=\beta_{1} \cdot \alpha_{1}=X
\end{array}
$$

and thus exhibits $X$ as a retract of $G Y_{2}$.

TheOrem 5.6. Suppose $\mathbf{G}_{1}, \mathbf{G}_{2}, \lambda$, $\mathbf{G}$ are as above. If $P\left(\mathbf{G}_{1}\right) \subset P\left(\mathbf{G}_{2}\right)$, then the $\mathbf{G}_{1}$ derived functors and the $\mathbf{G}$-derived functors are equivalent; if $P\left(\mathbf{G}_{2}\right) \subset P\left(\mathbf{G}_{1}\right)$, then the $\mathbf{G}_{2}$-derived functors and the $\mathbf{G}$-derived functors are equivalent.

Proof. The first condition implies that $P(\mathbf{G})=P\left(\mathbf{G}_{1}\right)$, while the second that $P(\mathbf{G})=$ $P\left(\mathbf{G}_{2}\right)$.

ProOF OF THEOREM 5.1. Let $\mathbf{G}_{z}$ denote the cotriple on the category of groups for which $G_{z} X=Z+X$ where $Z$ is the group of integers and + is the coproduct (free product). The augmentation and comultiplication are induced by the trivial map $Z \rightarrow 1$ and the "diagonal" map $Z \rightarrow Z+Z$ respectively. By the "diagonal" map $Z \rightarrow Z+Z$ is meant the map taking the generator of $Z$ to the product of the two generators of $Z+Z$. Map $Z \rightarrow G_{b f} Z$ by the map which takes the generator of $Z$ to the generator of $G_{b f} Z$ corresponding to it. For any $X$, map $G_{b f} Z \rightarrow G_{b f}(Z+X)$ by applying $G_{b f}$ to the coproduct inclusion. Also map $G_{b f} X \rightarrow G_{b f}(Z+X)$ by applying $G_{b f}$ to the other coproduct inclusion. Putting these together we have a map which is natural in $X$, $\lambda X: Z+G_{b f} X \rightarrow G_{b f}(Z+X)$, which can easily be seen to satisfy the data of a distributive law $G_{z} G_{b f} \longrightarrow G_{b f} G_{z}$. Also it is clear that $Z+G_{b f} X \simeq G_{f} X$, since the latter is free on exactly one more generator than $G_{b f} X$. Thus the theorem follows as soon as we observe that $P\left(\mathbf{G}_{z}\right) \supset P\left(\mathbf{G}_{b f}\right)$. In fact, the coordinate injection $\alpha: X \rightarrow Z+X$ is a map with $\varepsilon_{Z} \cdot \alpha=X$, and thus $P\left(\mathbf{G}_{z}\right)$ is the class of all objects.

Proof of theorem 5.2. It suffices to show that on $\mathfrak{M}, P\left(\mathbf{G}_{t}\right) \subset P\left(\mathbf{G}_{t}{ }^{\circ}{ }_{\lambda} \mathbf{G}_{k}\right)$. To do this, we factor $G_{t}=F_{t} U_{t}$ where $U_{t}: \mathfrak{M} \rightarrow \mathfrak{N}$, the category of $k$-projective $k$-modules, and $F_{t}$ is its coadjoint (the tensor algebra). For any $Y$, the map $U_{t} \varepsilon_{k} Y: U_{t} G_{k} Y \rightarrow U_{t} Y$ is easily seen to be onto, and since $U_{t} Y$ is $k$-projective, it splits, that is, there is a map $\gamma: U_{t} Y \rightarrow U_{t} G_{k} Y$ such that $U_{t} \varepsilon_{k} Y \cdot \gamma=U_{t} Y$. Then $G_{t} Y \stackrel{F_{t} \gamma}{\longrightarrow} G_{t} G_{k} Y \stackrel{G_{t} \varepsilon_{k} Y}{\longrightarrow} G_{t} Y$ presents any $G_{t} Y$ as a retract of $G_{t} G_{k} Y$. Clearly any retract of $G_{t} Y$ enjoys the same property.

The applicability of these results to other situations analogous to those of theorems 5.1 and 5.2 should be clear to the reader.

\section{Appendix}

In this appendix we give some of the more computational -and generally unenlighteningproofs so as to avoid interrupting the exposition in the body of the paper. 
A.1. Proof of Proposition 1.2. (1) When $n=i=0$ there is nothing to prove. If $i=0$ and $n>0$, we have by induction on $n$,

$$
\varepsilon^{n} \cdot \varepsilon^{m} G^{n}=\varepsilon \cdot \varepsilon^{n-1} G \cdot \varepsilon^{m} G^{n}=\varepsilon \cdot\left(\varepsilon^{n-1} \cdot \varepsilon^{m} G^{n-1}\right) G=\varepsilon \cdot \varepsilon^{n+m-1} G=\varepsilon^{n+m}
$$

If $i=n>0$, then we have by induction

$$
\varepsilon^{n} \cdot G^{n} \varepsilon^{m}=\varepsilon \cdot G \varepsilon^{n-1} \cdot G^{n} \varepsilon^{m}=\varepsilon \cdot G\left(\varepsilon^{n-1} \cdot G^{n-1} \varepsilon^{m}\right)=\varepsilon \cdot G \varepsilon^{n+m-1}=\varepsilon^{n+m}
$$

Finally, we have for $0<i<n$, again by induction,

$$
\varepsilon^{n} \cdot G^{i} \varepsilon^{m} G^{n-i}=\varepsilon^{i} \cdot G^{i} \varepsilon^{n-i} \cdot G^{i} \varepsilon^{m} G^{n-i}=\varepsilon^{i} \cdot G^{i} \varepsilon^{n+m-i}=\varepsilon^{n+m}
$$

(2) This proof follows the same pattern as in (1) and is left to the reader.

(3) When $n=0$ and $m=1$ these are the unitary laws. Then for $n=0$, we have, by induction on $m$,

$$
\begin{aligned}
G \varepsilon^{m} \cdot \delta^{m} & =G\left(\varepsilon \cdot \varepsilon^{m-1} G\right) \cdot \delta^{m}=G \varepsilon \cdot G \varepsilon^{m-1} G \cdot \delta^{m-1} G \cdot \delta \\
& =G \varepsilon \cdot\left(G \varepsilon^{m-1} \cdot \delta^{m-1}\right) G \cdot \delta=G \varepsilon \cdot \delta=G=\delta^{0}
\end{aligned}
$$

and similarly $\varepsilon^{m} G \cdot \delta^{m}=\delta^{0}$. Then for $n>0$, we have, for $i<n+1$,

$$
G^{n-i+1} \varepsilon^{m} G^{i} \cdot \delta^{n+m}=G^{n-i+1} \varepsilon^{m} G^{i} \cdot G^{n-i} \delta^{m} G^{i} \cdot \delta^{n}=G^{n-i}\left(G \varepsilon^{m} \cdot \delta^{m}\right) G^{i} \cdot \delta^{n}=\delta^{n}
$$

Finally, for $i=n+1$,

$$
\varepsilon^{m} G^{n+1} \cdot \delta^{n+m}=\varepsilon^{m} G^{n+1} \cdot \delta^{m} G^{n} \cdot \delta^{n}=\left(\varepsilon^{m} G \cdot \delta^{m}\right) G \cdot \delta^{n}=\delta^{n}
$$

(4) The proof follows the same pattern as in (3) and is left to the reader.

\section{A.2. Proof of theOrem 1.5.}

We must verify the seven identities which are to be satisfied by a simplicial homotopy. In what follows we drop most lower indices.

(1) $\varepsilon G^{n+1} d^{0} \cdot \delta G^{n} h^{0}=G^{n+1}\left(d^{0} \cdot h^{0}\right)=G^{n+1} \alpha_{n}$

(2) $G^{n+1} \varepsilon d^{n+1} \cdot G^{n} \delta h^{n}=G^{n+1}\left(d^{n+1} \cdot h^{n}\right)=G^{n+1} \beta_{n}$

(3) For $i<j$,

$$
\begin{aligned}
G^{i} \varepsilon G^{n+1-i} d^{i} & \cdot G^{j} \delta G^{n-j} h^{j}=G^{i}\left(\varepsilon G^{n+1-i} d^{i} \cdot G^{j-i} \delta G^{n-j} h^{j}\right) \\
& =G^{i}\left(G^{j-i-1} \delta G^{n-j} h^{j-1} \cdot \varepsilon G^{n-i} d^{i}\right)=G^{j-1} \delta G^{n-j} h^{j-1} \cdot G^{i} \varepsilon G^{n-i} d^{i}
\end{aligned}
$$

(4) For $0<i=j<n+1$,

$$
\begin{aligned}
G^{i} \varepsilon G^{n+1-i} d^{i} \cdot G^{i} \delta G^{n-i} h^{i} & =G^{n+1}\left(d^{i} \cdot h^{i}\right)=G^{n+1}\left(d^{i} \cdot h^{i-1}\right) \\
& =G^{i} \varepsilon G^{n+1-i} d^{i} \cdot G^{i-1} \delta G^{n-i+1} h^{i-1}
\end{aligned}
$$


(5) For $i>j+1$,

$$
\begin{aligned}
& G^{i} \varepsilon G^{n+1-i} d^{i} \cdot G^{j} \delta G^{n-j} h^{j}=G^{j}\left(G^{i-j} \varepsilon G^{n+1-i} d^{i} \cdot \delta G^{n-j} h^{j}\right) \\
& \quad=G^{j}\left(\delta G^{n-j-1} h^{j} \cdot G^{i-j-1} \varepsilon G^{n+1-i} d^{i-1}\right)=G^{j} \delta G^{n-j-1} h^{j} \cdot G^{i-1} \varepsilon G^{n+1-i} d^{i-1}
\end{aligned}
$$

(6) For $i \leq j$,

$$
\begin{aligned}
G^{i} \delta G^{n+1-i} s^{i} & \cdot G^{j} \delta G^{n-j} h^{j}=G^{i}\left(\delta G^{n+1-i} s^{i} \cdot G^{j-i} \delta G^{n-j} h^{j}\right) \\
& =G^{i}\left(G^{j-i+1} \delta G^{n-j} h^{j+1} \cdot \delta G^{n-i} s^{i}\right)=G^{j+1} \delta G^{n-j} h^{j+1} \cdot G^{i} \delta G^{n-i} s^{i}
\end{aligned}
$$

(7) For $i>j$,

$$
\begin{aligned}
& G^{i} \delta G^{n+1-i} s^{i} \cdot G^{j} \delta G^{n-j} h^{j}=G^{j}\left(G^{i-j} \delta G^{n+1-i} s^{i} \cdot \delta G^{n-j} h^{j}\right) \\
& \quad=G^{j}\left(\delta G^{n+1-j} h^{j} \cdot G^{i-1-j} \delta G^{n+1-i} s^{i-1}\right)=G^{j} \delta G^{n+1-j} h^{j} \cdot G^{i-1} \delta G^{n+1-i} s^{i-1}
\end{aligned}
$$

\section{A.3. Proof of THEOREM 1.6.}

We define $\alpha_{n}=\varepsilon^{n+1} X_{n}: G^{n+1} X_{n} \rightarrow X_{n}$ and $\beta_{n}=\delta^{n} X_{n} \cdot \vartheta X_{n}: X_{n} \rightarrow G^{n+1} X_{n}$. First we show that these are simplicial. We have

$$
d^{i} \cdot \alpha_{n}=d^{i} \cdot \varepsilon^{n+1} X_{n}=\varepsilon^{n+1} X_{n-1} \cdot d^{i}==\varepsilon^{n} X_{n} \cdot G^{i} \varepsilon G^{n-i} X_{n-1} \cdot G^{n+1} d^{i}=\alpha_{n} \cdot G^{i} \varepsilon G^{n-i} d^{i}
$$

Similarly,

$$
\begin{aligned}
s^{i} \cdot \alpha_{n}=s^{i} \cdot \varepsilon^{n+1} X_{n} & =\varepsilon^{n+1} X_{n+1} \cdot s^{i}=\varepsilon^{n+2} X_{n+1} \cdot G^{i} \delta G^{n-i} X_{n+1} \cdot s^{i}=\alpha_{n+1} \cdot G^{i} \delta G^{n-i} s^{i} \\
G^{i} \varepsilon G^{n-i} d^{i} \cdot \beta_{n} & =G^{i} \varepsilon G^{n-i} d^{i} \cdot \delta^{n} X_{n} \cdot \vartheta X_{n} \\
& =G^{n} d^{i} \cdot \delta^{n-i} X_{n} \cdot \vartheta X_{n}=\delta^{n-1} X_{n-1} \cdot \vartheta X_{n-1} \cdot d^{i}=\beta_{n-1} \cdot d^{i}
\end{aligned}
$$

Similarly,

$$
\begin{aligned}
G^{i} \delta G^{n-i} s^{i} \cdot \beta_{n} & =G^{i} \delta G^{n-i} s^{i} \cdot \delta^{n} X_{n} \cdot \vartheta X_{n}=\delta^{n+1} s^{i} \cdot \vartheta X_{n} \\
& =\delta^{n+1} X_{n+1} \cdot G s^{i} \cdot \vartheta X_{n}=\delta^{n+1} X_{n+1} \cdot \vartheta X_{n+1} \cdot s^{i}=\beta_{n+1} \cdot s^{i}
\end{aligned}
$$

Moreover, $\alpha_{n} \cdot \beta_{n}=\varepsilon^{n+1} X_{n} \cdot \delta^{n} X_{n} \cdot \vartheta X_{n}=\varepsilon X_{n} \cdot \vartheta X_{n}=X_{n}$.

Let $h_{n}^{i}=G^{i+1}\left(\delta^{n-i} s_{n}^{i} \cdot \vartheta X_{n} \cdot \varepsilon^{n-i} X_{n}\right): G^{n+1} X_{n} \rightarrow G^{n+2} X_{n+1}$ for $0 \leq i \leq n$. Then we will verify the identities which imply that $h: \beta \cdot \alpha \sim G^{*} X$. At most places in the computation below we will omit lower indices and the name of the objects under consideration.

$\varepsilon G^{n+1} d^{0} \cdot h_{n}^{0}=\varepsilon G^{n+1} d^{0} \cdot G\left(\delta^{n} s^{0} \cdot \vartheta \cdot \varepsilon^{n}\right)=\delta^{n}\left(d^{0} \cdot s^{0}\right) \cdot \vartheta \cdot \varepsilon^{n} \cdot \varepsilon G^{n}=\delta^{n} \cdot \vartheta \cdot \varepsilon^{n+1}=\beta_{n} \cdot \alpha_{n}$ 
(2)

$$
\begin{aligned}
G^{n+1} \varepsilon d^{n+1} \cdot h_{n}^{n} & =G^{n+1} \varepsilon d^{n+1} \cdot G^{n+1}\left(G s^{n} \cdot \vartheta\right)=G^{n+1}\left(\varepsilon d^{n+1} \cdot G s^{n} \cdot \vartheta\right) \\
& =G^{n+1}(\varepsilon \cdot \vartheta)=G^{n+1} X_{n}
\end{aligned}
$$

(3) For $i<j$,

$$
\begin{aligned}
G^{i} \varepsilon G^{n+1-i} d^{i} \cdot h_{n}^{j} & =G^{i} \varepsilon G^{n+1-i} d^{i} \cdot G^{j+1}\left(\delta^{n-j} s^{j} \cdot \vartheta \cdot \varepsilon^{n-j}\right) \\
& =G^{i}\left(\varepsilon G^{n+1-i} d^{i} \cdot G^{j-i+1}\left(\delta^{n-j} s^{j} \cdot \vartheta \cdot \varepsilon^{n-j}\right)\right) \\
& =G^{i}\left(G^{j-i}\left(\delta^{n-j} \cdot s^{j-1} \cdot \vartheta \cdot \varepsilon^{n-j}\right) \cdot \varepsilon G^{n-i} d^{i}\right) \\
& =G^{j}\left(\delta^{n-j} s^{j-1} \cdot \vartheta \cdot \varepsilon^{n-j}\right) \cdot G^{i} \varepsilon G^{n-i} d^{i}=h_{n-1}^{j-1} \cdot G^{i} \varepsilon G^{n-i} d^{i}
\end{aligned}
$$

(4) For $0<i=j<n+1$,

$$
\begin{aligned}
G^{i} \varepsilon G^{n+1-i} d^{i} \cdot h_{n}^{i} & =G^{i} \varepsilon G^{n+1-i} d^{i} \cdot G^{i+1}\left(\delta^{n-i} s^{i} \cdot \vartheta \cdot \varepsilon^{n-i}\right) \\
& =G^{i}\left(\varepsilon G^{n+1-i} d^{i} \cdot G\left(\delta^{n-i} s^{i} \cdot \vartheta \cdot \varepsilon^{n-i}\right)\right) \\
& =G^{i}\left(\delta^{n-i}\left(d^{i} \cdot s^{i}\right) \cdot \vartheta \cdot \varepsilon^{n-i} \cdot \varepsilon G^{n-i}\right) \\
& =G^{i}\left(\delta^{n-i} \cdot \vartheta \cdot \varepsilon^{n+1-i}\right)=G^{i}\left(\delta^{n-i}\left(d^{i} \cdot s^{i-1}\right) \cdot \vartheta \cdot \varepsilon^{n-i+1}\right) \\
& =G^{i}\left(\varepsilon G^{n+1-i} d^{i} \cdot \delta^{n-i+1} s^{i-1} \cdot \vartheta \cdot \varepsilon^{n-i+1}\right) \\
& =G^{i} \varepsilon G^{n+1-i} d^{i} \cdot G^{i}\left(\delta^{n-i+1} s^{i-1} \cdot \vartheta \cdot \varepsilon^{n-i+1}\right)=G^{i} \varepsilon G^{n+1-i} d^{i} \cdot h_{n}^{i-1}
\end{aligned}
$$

(5) For $i>j+1$,

$$
\begin{aligned}
G^{i} \varepsilon G^{n+1-i} d^{i} \cdot h_{n}^{j} & =G^{i} \varepsilon G^{n+1-i} d^{i} \cdot G^{j+1}\left(\delta^{n-j} s^{j} \cdot \vartheta \cdot \varepsilon^{n-j}\right) \\
& =G^{j+1}\left(G^{i-j-1} \varepsilon G^{n+1-i} d^{i} \cdot \delta^{n-j} s^{j} \cdot \vartheta \cdot \varepsilon^{n-j}\right) \\
& =G^{j+1}\left(\delta^{n-j-1}\left(d^{i} \cdot s^{j}\right) \cdot \vartheta \cdot \varepsilon^{n-j}\right) \\
& =G^{j+1}\left(\delta^{n-j-1}\left(s^{j} \cdot d^{i-1}\right) \cdot \vartheta \cdot \varepsilon^{n-j}\right) \\
& =G^{j+1}\left(\delta^{n-j-1} s^{j} \cdot \vartheta \cdot \varepsilon^{n-j-1} \cdot G^{i-1} \varepsilon G^{n-i+1} d^{i-1}\right) \\
& =h_{n-1}^{j} \cdot G^{i-1} \varepsilon G^{n-i+1} d^{i-1}
\end{aligned}
$$


(6) For $i \leq j$,

$$
\begin{aligned}
G^{i} \delta G^{n+1-i} s^{i} \cdot h_{n}^{j} & =G^{i} \delta G^{n+1-i} s^{i} \cdot G^{j+1}\left(\delta^{n-j} s^{j} \cdot \vartheta \cdot \varepsilon^{n-j}\right) \\
& =G^{i}\left(\delta G^{n+1-i} s^{i} \cdot G^{j-i+1}\left(\delta^{n-j} s^{j} \cdot \vartheta \cdot \varepsilon^{n-j}\right)\right) \\
& =G^{i}\left(G^{j-i+2}\left(\delta^{n-j} s^{j+1} \cdot \vartheta \cdot \varepsilon^{n-j}\right) \cdot \delta G^{n-i} s^{i}\right) \\
& =G^{j+2}\left(\delta^{n-j} s^{j+1} \cdot \vartheta \cdot \varepsilon^{n-j}\right) \cdot G^{i} \delta G^{n-i} s^{i}=h_{n+1}^{j+1} \cdot s^{i}
\end{aligned}
$$

(7) For $i>j$,

$$
\begin{aligned}
G^{i} \delta G^{n+1-i} s^{i} \cdot h_{n}^{j} & =G^{i} \delta G^{n+1-i} s^{i} \cdot G^{j+1}\left(\delta^{n-j} s^{j} \cdot \vartheta \cdot \varepsilon^{n-j}\right) \\
& =G^{j}\left(G^{i-j} \delta G^{n+1-i} s^{i} \cdot G \delta^{n-j} s^{j} \cdot G \vartheta \cdot G \varepsilon^{n-j}\right) \\
& =G^{j}\left(G \delta^{n-j+1}\left(s^{i} \cdot s^{j}\right) \cdot G \vartheta \cdot G \varepsilon^{n-j}\right) \\
& =G^{j}\left(G \delta^{n-j+1}\left(s^{j} \cdot s^{i-1}\right) \cdot G \vartheta \cdot G \varepsilon^{n-j}\right) \\
& =G^{j}\left(G \delta^{n-j+1} s^{j} \cdot G \vartheta \cdot G \varepsilon^{n-j} \cdot G^{i-j-1}(G \varepsilon \cdot \delta) G^{n+1-i} s^{i-1}\right) \\
& =G^{j}\left(G \delta^{n-j+1} s^{j} \cdot G \vartheta \cdot G \varepsilon^{n-j} \cdot G^{i-j} \varepsilon G^{n+1-i} \cdot G^{i-j-1} \delta G^{n+1-i} s^{i-1}\right) \\
& =G^{j}\left(G \delta^{n-j+1} s^{j} \cdot G \vartheta \cdot G \varepsilon^{n-j+1} \cdot G^{i-j-1} \delta G^{n+1-i} s^{i-1}\right) \\
& =G^{j+1}\left(\delta^{n-j+1} s^{j} \cdot \vartheta \cdot \varepsilon^{n-j+1}\right) \cdot G^{i-1} \delta G^{n+1-i} s^{i-1}=h_{n+1}^{j} \cdot G^{i-1} \delta G^{n+1-i} s^{i-1}
\end{aligned}
$$

This proof is adapted from the proof of Theorem 4.5 of [Appelgate (1965)].

\section{A.4. Proof of theorem 2.2.}

We must verify the three identities satisfied by a cotriple.

$$
\begin{aligned}
G \varepsilon \cdot \delta & =G_{1} G_{2} \varepsilon_{1} \varepsilon_{2} \cdot G_{1} \lambda G_{2} \cdot \delta_{1} \delta_{2}=G_{1} \varepsilon_{1} G_{2} \varepsilon_{2} \cdot \delta_{1} \delta_{2} \\
& =\left(G_{1} \varepsilon_{1} \cdot \delta_{1}\right)\left(G_{2} \varepsilon_{2} \cdot \delta_{2}\right)=G_{1} G_{2}=G
\end{aligned}
$$

$$
\begin{aligned}
\varepsilon G \cdot \delta & =\varepsilon_{1} \varepsilon_{2} G_{1} G_{2} \cdot G_{1} \lambda G_{2} \cdot \delta_{1} \delta_{2}=\varepsilon_{1} G_{1} \varepsilon_{2} G_{2} \cdot \delta_{2} \delta_{2} \\
& =\left(\varepsilon_{1} G_{1} \cdot \delta_{1}\right)\left(\varepsilon_{1} G_{2} \cdot \delta_{2}\right)=G_{1} G_{2}=G
\end{aligned}
$$

$$
\begin{aligned}
G \delta \cdot \delta & =G_{1} G_{2} G_{1} \lambda G_{2} \cdot G_{1} G_{2} \delta_{1} \delta_{2} \cdot G_{1} \lambda G_{2} \cdot \delta_{1} \delta_{2} \\
& =G_{1} G_{2} G_{1} \lambda G_{2} \cdot G_{1} \lambda G_{1} G_{2}^{2} \cdot G_{1}^{2} \lambda G_{2}^{2} \cdot G_{1} \delta_{1} G_{2} \delta_{2} \cdot \delta_{1} \delta_{2}=\lambda_{2} \cdot \delta_{1}^{2} \delta_{2}^{2}
\end{aligned}
$$

and by symmetry this latter is equal to $\delta G \cdot \delta$. 


\section{A.5. Proof of Proposition 2.4.}

(1) For $n=0$ this is vacuous and for $n=1$ it is an axiom. For $n>1$, we have by induction

$$
\begin{aligned}
G_{2}^{n} \varepsilon_{1} \cdot \lambda^{n} & =G_{2}^{n} \varepsilon_{1} \cdot G_{2} \lambda^{n-1} \cdot \lambda G_{2}^{n-1}=G_{2}\left(G_{2}^{n-1} \varepsilon_{1} \cdot \lambda^{n-1}\right) \cdot \lambda G_{2}^{n-1} \\
& =G_{2}\left(\varepsilon_{1} G_{2}^{n-1}\right) \cdot \lambda G_{2}^{n-1}=\left(G_{2} \varepsilon_{1} \cdot \lambda\right) G_{2}^{n-1}=\left(\varepsilon_{1} G_{2}\right) G_{2}^{n-1}=\varepsilon_{1} G_{2}^{n}
\end{aligned}
$$

(2) For $n=0$ this is vacuous and for $n=1$ it is an axiom. For $n>1$, we have by induction

$$
\begin{aligned}
G_{2}^{n} \delta_{1} \cdot \lambda^{n} & =G_{2}^{n} \delta_{1} \cdot G_{2} \lambda^{n-1} \cdot \lambda G_{2}^{n-1}=G_{2}\left(G_{2}^{n-1} \delta_{1} \cdot G_{2} \lambda^{n-1}\right) \cdot \lambda G_{2}^{n-1} \\
& =G_{2}\left(\lambda^{n-1} G_{1} \cdot G_{1} \lambda^{n-1} \cdot \delta_{1} G_{2}^{n-1}\right) \cdot \lambda G_{2}^{n-1} \\
& =G_{2} \lambda^{n-1} G_{1} \cdot G_{2} G_{1} \lambda^{n-1} \cdot\left(G_{2} \delta_{1} \cdot \lambda\right) G_{2}^{n-1} \\
& =G_{2} \lambda^{n-1} G_{1} \cdot G_{2} G_{1} \lambda^{n-1} \cdot\left(\lambda G_{1} \cdot G_{1} \lambda \cdot \delta_{1} G_{2}\right) G_{2}^{n-1} \\
& =G_{2} \lambda^{n-1} G_{1} \cdot G_{2} G_{1} \lambda^{n-1} \cdot \lambda G_{1} G_{2}^{n-1} \cdot G_{1} \lambda G_{2}^{n-1} \cdot \delta_{1} G_{2}^{n} \\
& =G_{2} \lambda^{n-1} G_{1} \cdot \lambda G_{2}^{n-1} G_{1} \cdot G_{1} G_{2} \lambda^{n-1} \cdot G_{1} \lambda G_{2}^{n-1} \cdot \delta_{1} G_{2}^{n}=\lambda^{n} G_{1} \cdot G_{1} \lambda^{n} \cdot \delta_{1} G_{2}^{n}
\end{aligned}
$$

(3) For $n=0$ this is an axiom. For $n>0$, first assume that $i=0$. Then we have by induction,

$$
\begin{aligned}
\varepsilon_{2} G_{2}^{n} G_{1} \cdot \lambda^{n+1} & =\varepsilon_{2} G_{2}^{n} G_{1} \cdot G_{2}^{n} \lambda \cdot \lambda^{n} G_{2}=G_{2}^{n-1} \lambda \cdot \varepsilon_{2} G_{2}^{n-1} G_{1} G_{2} \cdot \lambda^{n} G_{2} \\
& =G_{2}^{n-1} \lambda \cdot\left(\varepsilon_{2} G_{2}^{n-1} G_{1} \cdot \lambda^{n}\right) G_{2}=G_{2}^{n-1} \lambda \cdot\left(\lambda^{n-1} \cdot G_{1} \varepsilon_{2} G_{2}^{n-1}\right) G_{2} \\
& =G_{2}^{n-1} \lambda \cdot \lambda^{n-1} G_{2} \cdot G_{1} \varepsilon_{2} G_{2}^{n}=\lambda^{n} \cdot G_{1} \varepsilon_{2} G_{2}^{n}
\end{aligned}
$$

For $i>0$ we have, again by induction,

$$
\begin{aligned}
G_{2}^{i} \varepsilon_{2} G_{2}^{n-i} G_{1} \cdot \lambda^{n+1} & =G_{2}^{i} \varepsilon_{2} G_{2}^{n-i} G_{1} \cdot G_{2} \lambda^{n} \cdot \lambda G_{2}^{n}=G_{2}\left(G_{2}^{i-1} \varepsilon_{2} G_{2}^{n-i} G_{1} \cdot \lambda^{n}\right) \cdot \lambda G_{2}^{n} \\
& =G_{2}\left(\lambda^{n-1} \cdot G_{1} G_{2}^{i-1} \varepsilon_{2} G_{2}^{n-i}\right) \cdot \lambda G_{2}^{n}=G_{2} \lambda^{n-1} \cdot G_{2} G_{1} G_{2}^{i-1} \varepsilon_{2} G_{2}^{n-i} \cdot \lambda G_{2}^{n} \\
& =G_{2} \lambda^{n-1} \cdot \lambda G_{2}^{n-1} \cdot G_{1} G_{2}^{i} \varepsilon_{2} G_{2}^{n-i}=\lambda^{n} \cdot G_{1} G_{2}^{i} \varepsilon_{2} G_{2}^{n-i}
\end{aligned}
$$

(4) For $n=0$ this is an axiom. For $i=0$, we have by induction

$$
\begin{aligned}
\delta_{2} G_{2}^{n} G_{1} \cdot \lambda^{n+1} & =\delta_{2} G_{2}^{n} G_{1} \cdot G_{2}^{n} \lambda \cdot \lambda^{n} G_{2}=G_{2}^{n+1} \lambda \cdot \delta_{2} G_{2}^{n-1} G_{1} G_{2} \cdot \lambda^{n} G_{2} \\
& =G_{2}^{n+1} \lambda \cdot\left(\delta_{2} G_{2}^{n-1} G_{1} \cdot \lambda^{n}\right) G_{2}=G_{2}^{n+1} \lambda \cdot\left(\lambda^{n+1} \cdot G_{1} \delta_{2} G_{2}^{n-1}\right) G_{2} \\
& =G_{2}^{n+1} \lambda \cdot \lambda^{n+1} G_{2} \cdot G_{1} \delta_{2} G_{2}^{n}=\lambda^{n+2} \cdot G_{1} \delta_{2} G_{2}^{n}
\end{aligned}
$$


For $i>0$ we have, again by induction,

$$
\begin{aligned}
G_{2}^{i} \delta_{2} G_{2}^{n-i} G_{1} \cdot \lambda^{n+1} & =G_{2}^{i} \delta_{2} G_{2}^{n-i} G_{1} \cdot G_{2} \lambda^{n} \cdot \lambda G_{2}^{n}=G_{2}\left(G_{2}^{i-1} \delta_{2} G_{2}^{n-i} G_{1} \cdot \lambda^{n}\right) \cdot \lambda G_{2}^{n} \\
& =G_{2}\left(\lambda^{n+1} \cdot G_{1} G_{2}^{i-1} \delta_{2} G_{2}^{n-i}\right) \cdot \lambda G_{2}^{n}=G_{2} \lambda^{n+1} \cdot G_{2} G_{1} G_{2}^{i-1} \delta_{2} G_{2}^{n-i} \cdot \lambda G_{2}^{n} \\
& =G_{2} \lambda^{n+1} \cdot \lambda G_{2}^{n+1} \cdot G_{1} G_{2}^{i} \delta_{2} G_{2}^{n-i}=\lambda^{n+2} \cdot G_{1} G_{2}^{i} \delta_{2} G_{2}^{n-i}
\end{aligned}
$$

A.6. Proof of Proposition 4.1 (3).

In the following we let $d^{i}$ and $s^{i}$ stand for $d^{i} X$ and $s^{i} X$ respectively. If $Y=G_{p} X$, then $Y_{n}=X_{n+1}, d^{i} Y=d^{i+1}$ and $s^{i} Y=s^{i+1} . \alpha_{n}=\left(d^{1}\right)^{n+1}: Y_{n} \rightarrow X_{0}$ and $\beta_{n}=$ $\left(s^{0}\right)^{n+1}: X_{0} \rightarrow Y_{n}$. Then $\alpha_{n} \cdot \beta_{n}=\left(d^{1}\right)^{n+1} \cdot\left(s^{0}\right)^{n+1}=Y_{n}$. Let $h_{n}^{i}=\left(s^{0}\right)^{i+1}\left(d^{1}\right)^{i}: Y_{n} \rightarrow Y_{n+1}$ for $0 \leq i \leq n$.

(1) $d^{0} Y \cdot h^{0}=d^{1} \cdot s^{0}=Y_{n}$.

(2) $d^{n+1} Y \cdot h^{n}=d^{n+2} \cdot\left(s^{0}\right)^{n+1} \cdot\left(d^{1}\right)^{n}=\left(s^{0}\right)^{n+1} \cdot d^{1} \cdot\left(d^{1}\right)^{n}=\beta_{n} \cdot \alpha_{n}$.

(3) For $i<j$,

$$
\begin{aligned}
d^{i} Y \cdot h^{j} & =d^{i+1} \cdot\left(s^{0}\right)^{j+1} \cdot\left(d^{1}\right)^{j}=\left(s^{0}\right)^{j} \cdot d^{i} \cdot\left(d^{1}\right)^{j} \\
& =\left(s^{0}\right)^{j} \cdot\left(d^{1}\right)^{j-1} \cdot d^{i+1}=h^{j-1} \cdot d^{i} Y
\end{aligned}
$$

$$
\begin{aligned}
d^{i} Y \cdot h^{i} & =d^{i+1} \cdot\left(s^{0}\right)^{i+1} \cdot\left(d^{1}\right)^{i}=\left(s^{0}\right)^{i} \cdot\left(d^{1}\right)^{i} \\
& =\left(s^{0}\right)^{i} \cdot d^{1} \cdot\left(d^{1}\right)^{i-1}=d^{i+1} \cdot\left(s^{0}\right)^{i} \cdot\left(d^{1}\right)^{i-1}=d^{i} Y \cdot h^{i-1}
\end{aligned}
$$

(5) For $i>j+1$,

$$
\begin{aligned}
d^{i} Y \cdot h^{j} & =d^{i+1} \cdot\left(s^{0}\right)^{j+1} \cdot\left(d^{1}\right)^{j}=\left(s^{0}\right)^{j+1} \cdot d^{i-j} \cdot\left(d^{1}\right)^{j} \\
& =\left(s^{0}\right)^{j+1} \cdot\left(d^{1}\right)^{j} \cdot d^{i}=h^{j} \cdot d^{i-1} Y
\end{aligned}
$$

(6) For $i \leq j$,

$$
\begin{aligned}
s^{i} Y \cdot h^{j} & =s^{i+1} \cdot\left(s^{0}\right)^{j+1} \cdot\left(d^{1}\right)^{j}=\left(s^{0}\right)^{j+2} \cdot\left(d^{1}\right)^{j} \\
& =\left(s^{0}\right)^{j+2} \cdot\left(d^{1}\right)^{j+1} \cdot s^{i+1}=h^{j+1} \cdot s^{i} Y
\end{aligned}
$$

(7) For $i>j$,

$$
\begin{aligned}
s^{i} Y \cdot h^{j} & =s^{i+1} \cdot\left(s^{0}\right)^{j+1} \cdot\left(d^{1}\right)^{j}=\left(s^{0}\right)^{j+1} \cdot s^{i-j} \cdot\left(d^{1}\right)^{j} \\
& =\left(s^{0}\right)^{j+1} \cdot\left(d^{1}\right)^{j} \cdot s^{i}=h^{j} \cdot s^{i-1} Y
\end{aligned}
$$

\section{A.7. Proof of Proposition 4.4.}

Form the double simplicial object $E=\left\{E_{i j}=G_{t}^{i+1} X_{j}\right\}$ with the maps gotten by applying $G$ to the faces and degeneracies of $X$ in one direction and the cotriple faces and degeneracies in the other. Let $D=\left\{D_{i}=G_{t}^{i+1} X_{i}\right\}$ be the diagonal complex. We are trying to show that $\pi_{0} D \simeq \pi_{0} X$. But the Dold-Puppe theorem asserts that $\pi_{0} D \simeq H_{0} N D$ 
and the Eilenberg-Zilber theorem asserts that $H_{0} N D$ is $H_{0}$ of the total complex associated with $E$. But we may compute the zero homology of

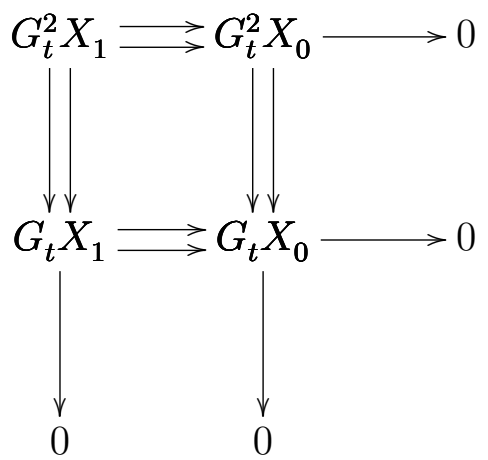

by first computing the 0 homology vertically, which gives, by another application of the Dold-Puppe theorem,

$$
\pi_{0}\left(G_{t}^{*} X_{1}\right) \Longrightarrow \pi_{0}\left(G_{t}^{*} X_{0}\right) \longrightarrow 0
$$

But $G_{t}^{*}$ is readily shown to be right exact (i.e. it preserves coequalizers) and so this is $X_{1} \longrightarrow X_{0} \longrightarrow 0$. Another application of the Dold-Puppe theorem gives that $H_{0}$ of this is $\pi_{0} X$.

\section{References}

[Appelgate (1965)] H. Appelgate, Acyclic models and resolvent functors. Dissertation, Columbia University, New York (1965).

[Barr (1966)] M. Barr, Cohomology in tensored categories. Proc. Conf. Categorical Algebra (La Jolla, 1965). Springer-Verlag, Berlin (1966), 344-354.

[Barr (1967)] M. Barr, Shukla cohomology and triples. J. Alg. 5 (1967), 222-231.

[Barr \& Beck (1966)] M. Barr \& J. Beck, Acyclic models and triples. Proc. Conf. Categorical Algebra (La Jolla, 1965). Springer-Verlag, Berlin (1966), 336-343.

[Barr \& Beck (1969)] M. Barr \& J. Beck, Homology and standard constructions. Lecture Notes Math. 80. Reprinted in Theorem and Applications of Categories, 18 (1969), http://www.tac.mta.ca/ tac/reprints/articles/18/tr18.pdf .

[Beck (1967)] J. Beck, Triples, algebras, and cohomology. Dissertation, Columbia University, New York (1967). Republished electronically, Reprinted as Theory and Applications of Categories, 2 (2003), http://www.tac.mta.ca/tac/reprints/articles/2/tr2abs.html

[Dold \& Puppe (1961)] A. Dold \& D. Puppe, Homologie nicht-additiver Funktoren. Anwendungen. Ann. Inst. Fourier 11 (1961), 201-312.

[Huber (1961)] P. J. Huber, Homotopy theory in general categories. Math. Ann. 144 (1961), 361-385.

[Mac Lane (1963)] S. Mac Lane, Homology. Springer-Verlag, Berlin (1963).

[Shukla (1961)] U. Shukla, Cohomologie des algèbres associatives. Ann. Sci. École Norm. Sup. 78 (1961), 163-209. 